\title{
Evaluation of EGM2008 by means of GPS Levelling Uganda
}

\author{
Dianah Rose $\mathrm{Abeho}^{1}$, Roger Hipkin ${ }^{2}$ and Bedada Besha Tulu ${ }^{3}$ \\ ${ }^{1}$ Geomatics and Land Management Department, Makerere University, Kampala, Uganda, \\ dabeho@tech.mak.ac.ug \\ ${ }^{2}$ School of GeoSciences, University of Edinburgh, UK \\ ${ }^{3}$ Institute of Geophysics, Space Sciences and Astronomy, Addis Ababa University, Addis Ababa, \\ Ethiopia
}

DOI: http://dx.doi.org/10.4314/sajg.v3i3.2

\begin{abstract}
The global gravity model EGM2008 is evaluated in various regions of Uganda to assess if it is good enough for geodetic applications. The evaluation method involves comparison of geoid heights computed from the model with those computed at irregularly distributed GPS Levelling stations. For testing the model, a total of seven levelled benchmarks available in Uganda which belong to the New Khartoum datum were used. The spatial positions of these benchmarks were determined at $\mathrm{mm}$ accuracy, with respect to ITRF2008. The agreement between the EGM2008 geoid and the geoid undulation derived from GPS Levelling over the seven irregularly distributed benchmark points has a standard deviation of $0.255 \mathrm{~m}$, with a mean of $-0.859 \mathrm{~m}$. The datum offset may be due the choice of Wo (potential of the geoid) and Uo (potential on the surface of the ellipsoid); using GRS80 for the gravitational reference system and WGS84 for the geometrical reference system; some possibly different tidal conventions; but, by using the same method of analysis for Ethiopia and Uganda, these absolute offset effects are eliminated when comparing the two so that the computed difference $[0.118 \mathrm{~m}]$ in datum offset for the two states does tell us something about the differences in levelling datums. The standard deviation of $0.255 \mathrm{~m}$ suggests that sparser, irregularly-distributed and inhomogenous gravity data for Uganda was used in the development of EGM2008 not ruling out errors in levelling since there is barely any documentation pertaining the accuracy of results obtained regarding the levelling network in Uganda.
\end{abstract}

\section{Introduction}

Most geodetic applications like determining the topographic heights of points on the globe require the geoid which approximates Mean Sea Level (MSL), as the datum/reference surface. In this satellite era one is capable of obtaining a sufficiently accurate model of the gravity field over the surface of the earth. This is a great achievement in the fields of geodesy and geophysics since we can achieve heights with physical meaning without necessarily carrying out the tedious and time-consuming procedures of obtaining these heights by geometric or trigonometric levelling. 
The release of the new Earth Gravitational Model EGM2008 by the US National Geospatial Intelligence Agency (Pavlis et al., 2008) is a big achievement in determination of the earth's gravity field. EGM2008 is a spherical harmonic model complete to degree and order of 2159, with additional spherical harmonic coefficients extending up to degree of 2190 and order 2159. This offers a spatial sampling resolution of approximately $9 \mathrm{~km}$ for the recovery of gravity field functions over the entire globe (Kotsakis et al., 2008). EGM2008 incorporates $5 \times 5$ min gravity anomalies and altimetry-derived gravity anomalies and has benefited from the latest GRACE based satellite gravity field model (Pavlis et al., 2008).

For this model to be used for geodetic activities anywhere on the globe, there is need to quantify its accuracy using several validation data sets such as geoid heights through the combination of GPS and levelling, airborne and local surface gravity measurements, marine geoid heights from mean oceanographic surface topography models and altimetry observations among others (Kotsakis et al., 2008; Kılıçoğlu et al., 2008). For better assessment, however, these external data sets should be independent of the estimation procedures that were used in the development of the model.

This study focuses on assessing the accuracy of the EGM2008 model in various regions of Uganda using GPS Levelling over a network of seven points that exhibit long wave length structures in the levelling heights. If this model best fits the study area, it could further be applied in determination of the geoid at both regional and national scales.

\section{Heighting systems}

\section{Orthometric heights}

Orthometric heights $(\mathrm{H})$ refer to an equipotential reference surface such as the geoid. The orthometric height of a distinct point on the surface of the earth is the distance from that point to the geoid, measured along the plumb line normal to the geoid (Heister et al., 1999). Due to the fact that equipotential surfaces are not parallel, this plumb line is a curved line. Orthometric heights can be derived using various methods including spirit or trigonometric levelling, global gravity models constituted from potential coefficients, use of vertical deflection obtained from astro-geodetic measurement and gravimetric measurement among others.

\section{Ellipsoidal heights}

Ellipsoidal heights $(h)$ refer to a reference ellipsoid such as the WGS84. The height $h$ of a point is defined as the distance from the ellipsoid measured along a normal to the reference ellipsoid. Ellipsoidal heights can be derived from geocentric Cartesian coordinates provided by GPS observations (Heister et al., 1999). The difference between orthometric heights and ellipsoidal 
heights is defined as the geoid height $(N)$.The basic relationship between geoid, ellipsoidal and orthometric heights is given by the following equation:

$$
h=H+N
$$

$h$ is the ellipsoidal height computed from GPS and $H$ is the orthometric height computed from levelling.

\subsection{Methods of determining orthometric heights}

\subsubsection{Geometric levelling}

Geometric levelling is the determination of the height differences by using a level and a vertical rod. Geometric levelling may at first appear as a very simple method and one that yields the best result. However, practical applications have shown that carrying out this method is very difficult on rough ground and is sensitive to regular or irregular model errors. Preventative measures must be taken to eliminate or reduce model errors stemming from instrumental and ambient conditions. They decrease the speed of survey, thus the cost of surveying rises (Banger, 1981; Niemeier, 1986; Ceylan,1988; Baykal, 1989).

\subsubsection{Trigonometric levelling}

In trigonometric levelling, the height differences are computed by using vertical angle and slope distance. Depending on the type of terrain, time available and which vertical angles are observed, trigonometric levelling can be classified into three categories (Rueger and Brunner, 1982; Kuntz and Schmitt, 1986; Aksoy et al., 1993):

a) Unidirectional trigonometric levelling

b) Leap-Frog (jumped) trigonometric levelling

c) Reciprocal trigonometric levelling

\subsubsection{GPS Levelling}

GPS Levelling is the most recent and advanced method that can be used in the determination of heights. Three-dimensional coordinates can be obtained by GPS in the geocentric Cartesian coordinate system. The Cartesian coordinates are transformed to geodetic latitude $\left({ }^{\varphi}\right)$, geodetic longitude (2), and ellipsoidal heights ( $h$ ) according to selected reference ellipsoid, such as WGS84. The ellipsoidal heights obtained by GPS are not directly used for practical surveying, but can be transformed to orthometric heights. However geoid heights of all points have to be computed before computing orthometric heights. 
Classically orthometric heights were computed from a combination of gravity measurements with height increments. Geometric levelling sums up the height increments, but ignores gravity. As such it will give an apparent height difference that will depend on the path taken and when carried out with very high precision, levelling around a closed loop will not close. A proper height system must explicitly determine the difference in potential (geopotential number) by summing the height increments multiplied by a measured gravity value:

$$
C_{p}=W_{o}-W_{p}=\sum_{0}^{P}\left|g_{i}\right| \Delta H_{i}
$$

Where:

$\mathrm{Cp}$ is the geopotential number at a particular point $(\mathrm{P})$

$\mathrm{W}_{\mathrm{o}}$ is the potential at the zero datum

$\mathrm{W}_{\mathrm{p}}$ is the gravitational potential energy and depends only on where point $\mathbf{P}$ is, it doesn't depend on the route taken by the leveling.

$\mathrm{g}_{\mathrm{i}}$ is the measured gravity at each respective station

$\Delta \mathrm{H}$ is the height increment at each respective station

A geometrical conversion could be found by dividing this geopotential number by a constant gravity value $(g)$,

$$
H_{P}=\frac{C_{P}}{\bar{g}_{P}}
$$

Where:

$\mathrm{Hp}$ is the orthometric height at point $\mathrm{P}$

$\bar{g}_{P}$ is the average gravity along the plumb line

$\mathrm{Cp}$ is the geopotential number at $\mathrm{P}$

The logical difficulty with this approach is that it needs $\bar{g}_{P}$, an average gravity value inside the earth. In principle, we cannot know this value, but ways of estimating it have been devised. A better approach of determining orthometric height in the satellite era is the use of global geopetential models. Using GPS, an absolute height above a properly geocentric ellipsoid can be obtained. Geopotential heights (from which orthometric height above sea level could be derived) can be obtained by inserting the coordinates given by the satellite positioning $(h, \varphi, \lambda)$ into a global model of the earth's potential. 


\section{Study Area and Data Set}

The study area is Uganda, located in eastern part of Africa and on the north-western shores of lake Victoria, extending from $1^{\circ}$ South to $4^{\circ}$ North latitude and $30^{\circ}$ to $35^{\circ}$ East longitude defining a total area of 241,139 square kilometres.

This study involves a network of seven unevenly distributed fundamental benchmarks in Uganda, which were established by the Directorate of Overseas Surveys (DOS) for the Surveys and Mapping Department (SMD) in Entebbe, Uganda. These seven benchmark points are unevenly distributed with the majority of the points in the south western region of Uganda. This is because some of the fundamental benchmark points earlier established were either destroyed or the description cards that define the location of the benchmarks refer to features that are temporary like semi-permanent building structures and no longer exist. This made it hard to locate sufficient benchmark points that give a good representation of Uganda as a country. These fundamental benchmarks refer to the Egyptian Benchmark BM 9029, itself related to MSL Alexandria. Figure 1 shows the distribution of the seven benchmarks.

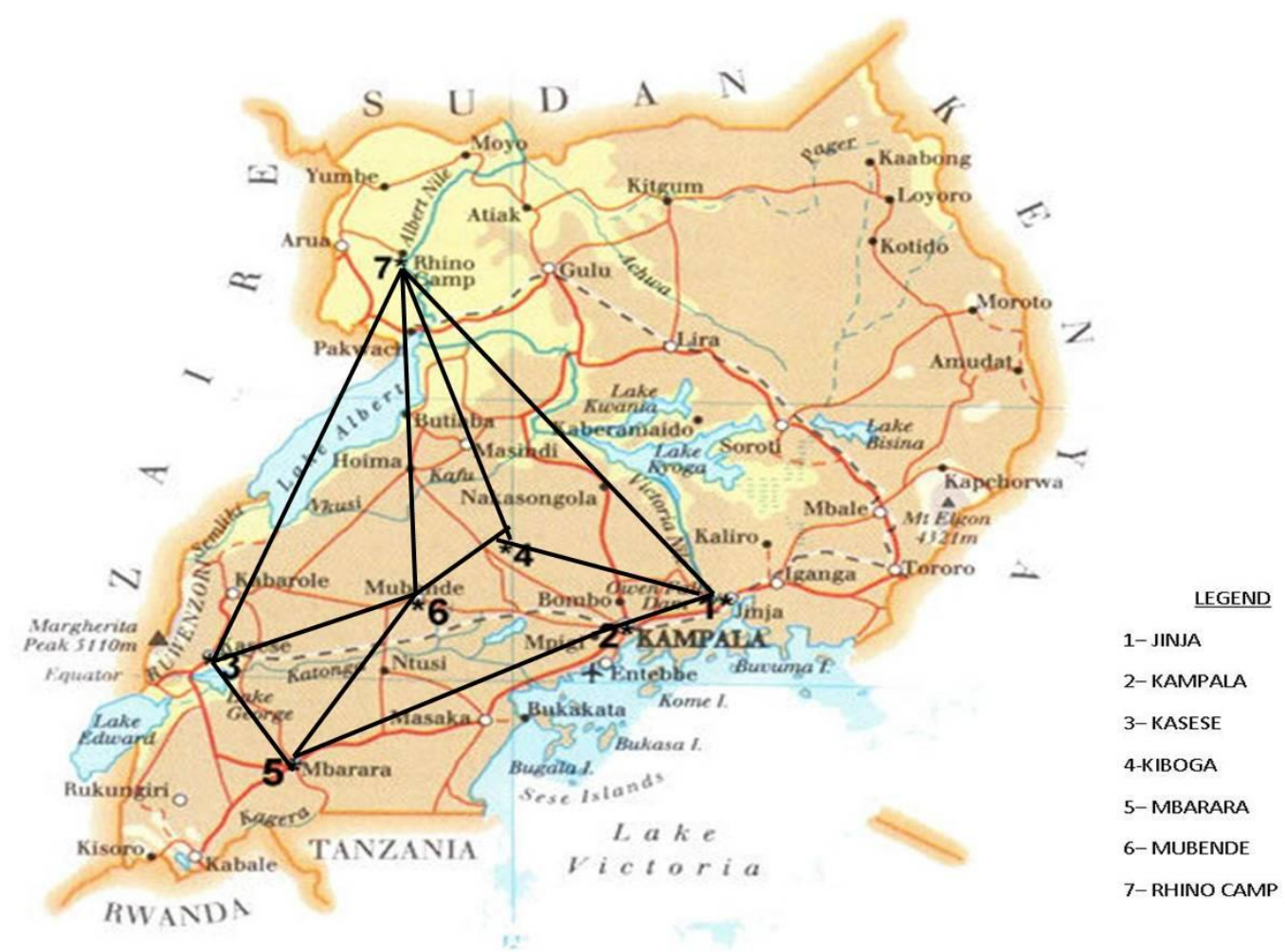

Figure 1. Geographical Distribution of the benchmark points in Uganda (http://www.mapcruzin.com)

\section{Ellipsoidal heights}

Ellipsoidal heights of the network points were determined by making static GNSS observations using dual frequency Trimble R7 GNSS receivers with respect to the IGS realisation of the ITRF 2008 reference frame. A GNSS campaign was undertaken starting $10^{\text {th }}$ February to $18^{\text {th }}$ February, 
2012 and observations were made on the respective benchmarks for a minimum of 48 hours and a maximum of 144 hours.

To ensure that the GNNS equipment was in proper condition, GNSS observations were made on known control points, the coordinates computed and compared with the known. Other Strategies put in place to minimise errors in the coordinates as a result poor GNNS observations include; long observation time, use of the same type of receivers for all the observations, checking of the instrument heights hourly, use of Trimble Zephyr Geodetic Antenna. The Zephyr Geodetic Antenna's quality performance and extreme accuracy are achieved through sub-millimeter phase center repeatability, robust low-elevation tracking and significantly reduced ground-based multipath (http://www.dpie.com/gps/trimble-zephyr-geodetic-gnss-antenna.html).

Table 1 below presents the receiver details and occupation times for each of the seven benchmark points.

Table 1. GNSS occupation times and receiver details

\begin{tabular}{lll}
\hline Station & & Occupation time (hours) \\
\hline JINJA & 144 & Receiver type \\
71Y97 (Kampala) & 48 & Trimble R7 GNSS \\
KASESE & 48 & Trimble R7 GNSS \\
KIBOGA & 48 & Trimble R7 GNSS \\
MBARARA & 144 & Trimble R7 GNSS \\
MUBENDE & 48 & Trimble R7 GNSS \\
RHINO CAMP & 48 & Trimble R7 GNSS \\
\hline
\end{tabular}

\section{Levelled Heights}

The results of the precise leveling were obtained from the Surveys and Mapping Department in Entebbe.The levelled heights of the fundamental benchmarks in Uganda refer to the MSL at Alexandria. Before the second world war, the Directorate of Overseas Surveys carried out precise differential levelling from the Kenyan coast to Uganda. As the reference sea level for this exercise was the Mombasa tide gauge, the datum was called MSL Mombasa. Another differential levelling came into Uganda all the way from Egypt (Alexandria) through Sudan. The reference sea level was obtained from the harbour of Alexandria, and new calculations for the altitude of all the benchmarks were carried out, in the so-called New Khartoum datum (the fundamental point of which is given at 363.082m above MSL at Alexandria) and consequently tied to the new MSL Alexandria datum. A block adjustment was carried out by the Survey Department using observation equation method, producing values referring to MSL Alexandria with a standard error of $0.00035 \mathrm{~m}$ per unit weight. The first order network was completed by 1970, but the second order network was done only in some limited areas of the country and around Lake Kyoga before the start of the political turmoil. The difference between the new datum and the old one was calculated as $-0.01676 \mathrm{~m}$ (MSL 
Mombassa is $0.01676 \mathrm{~m}$ lower than MSL Alexandria at the Khartoum gauge). Thus the altitudes in Uganda are based on only one connection to the Egyptian Benchmark BM 9029, itself related to MSL Alexandria. And therefore the heights of the benchmarks in this study are refrenced to the same Benchmark BM 9029.

\section{GPS processing}

The Receiver INdependent EXchange (Rinex) data for each of the benchmark stations was processed using GAMIT/GLOBK software based on the realisation of the ITRF 2008 reference frame using IGS orbit product precise ephemeris.

GAMIT is a collection of programs to process GPS phase data to estimate three-dimensional relative positions of ground stations and satellite orbits, atmospheric zenith delays, and earth orientation parameters (http://www-gpsg.mit.edu). To control processing, the software uses C-shell scripts which invoke the Fortran or $\mathrm{C}$ programs compiled in the respective directories. The software is designed to run under any UNIX operating system. The maximum number of stations and atmospheric parameters allowed is determined by dimensions set at compile time and can be tailored to fit the requirements and capabilities of the analyst's computational environment.

GLOBK is a Kalman filter whose primary purpose is to combine various geodetic solutions such as GPS, VLBI, and SLR experiments. It accepts the estimates and covariance matrices for station coordinates, earth-orientation parameters, orbital parameters, and source positions generated from the analysis of the primary observations as inputs (http://www-gpsg.mit.edu). GLOBK was used to combine the individual sessions (days) of observations to obtain an estimate of station coordinates averaged over a 3-6 day experiment.

The coordinates for each of the benchmark points on the respective days were computed to mm accuracy both in the North, East and Up (NEU) components with reference to WGS84 reference ellipsoid. All the coordinates were computed relative to three IGS network stations in Addis Ababa (ADIS), South Africa (HARB) and GABON (NKLG). The stations were chosen based on proximity to the local network and stability based on the velocity solutions as presented in ITRF08. By comparing different solutions using several IGS stations in Africa, the above three IGS stations yielded the best results and were therefore adopted for the processing.

GLOBK was used to compute the average of the daily solutions obtained using GAMIT. Table 2 shows the computed geodetic coordinates for the benchmarks. 
Table 2. Geodetic coordinates for the benchmark points and their levelled heights

\begin{tabular}{|c|c|c|c|c|}
\hline Station & Latitude (DMS) & Longitude (DMS) & $\begin{array}{l}\text { Ellipsoidal } \\
\text { Height (m) }\end{array}$ & $\begin{array}{c}\text { Levelled } \\
\text { Height (m) }\end{array}$ \\
\hline JINJA & $0^{\circ} 25^{\prime} 08.69758^{\prime \prime}$ & $33^{\circ} 12^{\prime} 00.39103^{\prime \prime}$ & 1162.498 & 1176.064 \\
\hline 71 Y97 (Kampala) & $0^{\circ} 20^{\prime} 17.73450 "$ & $32^{\circ} 33^{\prime} 53.31548^{\prime \prime}$ & 1255.314 & 1267.942 \\
\hline KASESE & $0^{\circ} 10^{\prime} 46.06511^{\prime \prime}$ & $30^{\circ} 04^{\prime} 37.67715^{\prime \prime}$ & 980.896 & 990.380 \\
\hline KIBOGA & $0^{\circ} 54^{\prime} 52.41815^{\prime \prime}$ & $31^{\circ} 46^{\prime} 09.67010^{\prime \prime}$ & 1174.464 & 1187.649 \\
\hline MBARARA & $-0^{\circ} 36^{\prime} 33.68073^{\prime \prime}$ & $30^{\circ} 39^{\prime} 12.62674 "$ & 1448.208 & 1459.021 \\
\hline MUBENDE & $0^{\circ} 33^{\prime} 43.59150^{\prime \prime}$ & $31^{\circ} 23^{\prime} 24.85620^{\prime \prime}$ & 1311.438 & 1324.002 \\
\hline RHINO CAMP & $2^{\circ} 58$ ' 17.78812” & $31^{\circ} 23^{\prime} 43.05879^{\prime \prime}$ & 617.809 & 631.387 \\
\hline
\end{tabular}

Table 3 presents the Normalised Root Mean Square (NRMS) and Weighted Root Mean Square (WRMS) of the NEU coordinates extracted from the NEU residual plots.

Table 3. Root Mean Square Error of the Computed coordinates

\begin{tabular}{lcccccc}
\hline \multirow{2}{*}{ Station } & \multicolumn{2}{c}{ Northings $(\mathbf{m m})$} & \multicolumn{2}{c}{ Eastings $(\mathbf{m m})$} & \multicolumn{2}{c}{ Up (mm) } \\
\cline { 2 - 7 } & NRMS & WRMS & NRMS & WRMS & NRMS & WRMS \\
\hline JINJA & 0.74 & 3.1 & 0.58 & 3.4 & 0.33 & 7.3 \\
KAMPALA & 0.73 & 2.4 & 0.10 & 0.4 & 0.22 & 3.2 \\
KASESE & 0.28 & 1.7 & 0.03 & 0.3 & 0.12 & 4.3 \\
KIBOGA & 0.41 & 1.3 & 0.07 & 0.3 & 0.40 & 5.6 \\
MBARARA & 1.02 & 2.6 & 0.60 & 1.8 & 0.41 & 4.1 \\
MUBENDE & 0.10 & 0.7 & 0.69 & 4.0 & 0.35 & 9.4 \\
RHINO CAMP & 0.10 & 0.3 & 0.47 & 1.6 & 0.84 & 9.5 \\
\hline
\end{tabular}

\section{Results}

\section{GPS-based geiod heights}

The geoid heights at the seven points were computed according to equation (4) based on the computed ellipsoidal and known levelled heights.

$$
N^{G P S / l e v}=h-H
$$

Where $\mathrm{N}^{\text {GPS/lev }}$ is the geoid height determined by GPS Levelling. $\mathrm{N}^{\text {GPS/lev }}$ provides the dataset upon which the evaluation tests are performed. 


\section{EGM2008-based geiod heights}

The Synthesis FORTRAN program, Tg_Schmidt.f together with the coefficients of EGM2008 global gravity model adjusted to Schmidt semi-normalisation, was used to compute the anomalous potential and gravity disturbance on a regular grid of latitude and longitude covering the whole region of Uganda and at nine levels with a vertical spacing of 500m on and above the ellipsoid. The Tg_Schmidt.f program was initially written by Dr Roger Hipkin in 1972 with updates added over the past 40 years. This programme uses basic formulae for the space domain spherical harmonic representations of the earth's potential or gravity.

Using the interpolation and height comparison FORTRAN program, Tg_interp.f, together with the binary files output by Tg_Schmidt.f, values of the geoid height and hence orthometric height $H$ are obtained at each of the surveyed stations.

The ellipsoidal heights $h$ provided by GPS allow gravity disturbance and anomalous potential to be estimated both on the ellipsoid and on the ground surface, and continuously at all points in between. This can then give an average value for gravity disturbance between the ellipsoid and the ground surface. Normal gravity and the reference potential can be evaluated similarly using standard formulae. The combination of the gravity disturbance and normal gravity gives 'free-air gravity', $g^{*}$, which is subterranean gravity that ignores the attraction of the topographic masses, but is analytically continuous with the external gravity field and the information used to construct EGM2008.

If we neglect the term in the square of anomalous gravity, the integrals relating distance and force to potential energy become:

$$
\overline{\mathrm{g}} * h=\int_{h=0}^{h=h} \mathrm{~g}^{*}(h) d h=W(0)-W(h)
$$

Where $\mathrm{W}(0)$ is the real potential of the earth on the reference ellipsoid and $W(h)$ is the real potential of the earth at the surface point ' $h$ '.

Similarly

$$
\overline{\mathrm{g}}^{*} H=\int_{h=N}^{h=h} \mathrm{~g}^{*}(h) d h=W(N)-W(H)
$$

Where $\mathrm{W}(\mathrm{N})=\mathrm{W}_{0}$ is a real potential of the earth evaluated on the geoid/ the potential of the geoid and $\mathrm{W}(\mathrm{H})$ is the real potential of the earth at the surface point ' $\mathrm{H}$ ' whereas $W_{\mathrm{o}}=62636854.19 \mathrm{~m}^{2} \mathrm{~s}^{-2}$ is the real earth potential at mean sea level (Dayoub et al. 2012).

After expanding the integral in equation 5 based on equation 1, and subtracting equation 6 from the new form of equation 5 , one can get the following;

$$
h=H+\frac{W(0)-W(N)}{\overline{\mathrm{g}}^{*}}
$$


An expansion of the real earth potential at a small height $\Delta h$ above the ellipsoid gives

$$
W(\Delta h)=W(0)+\frac{\partial W(0)}{\partial h} \Delta h+\cdots
$$

so, putting $\Delta h=N$,

$$
\frac{W(0)-W(N)}{\overline{\mathrm{g}}^{*}} \approx \frac{-\frac{\partial W(0)}{c h}}{\overline{\mathrm{g}}^{*}} \approx N
$$

from which

$$
h=H+N
$$

The expression duplicates a classical result, but does not indicate how $N$ can be calculated from a program that calculates the anomalous potential, $T$.

$$
N=\frac{W(0)-W(N)}{\overline{\mathrm{g}}^{*}}=\frac{U(0)+T(0)-W(N)}{\overline{\mathrm{g}}^{*}}=\frac{U_{O}-W_{O}+T(0)}{\overline{\mathrm{g}}^{*}}
$$

Note that $T(0)$ is the anomalous potential on the reference ellipsoid, $\mathrm{U}(0)=\mathrm{U}_{0}$ is a normal potential on the reference ellipsoid and it is computed from four geodetic parameters namely equatorial radius, second degree zonal harmonic of the potential, geocentric gravitational constant and angular rotation rate of the earth's spin. $\mathrm{U}(0)=\mathrm{U}_{0}=62636860.85$ is the computed normal potential on GSR80 reference ellipsoid. Thus $N$, the height of the levelling datum, essentially involves the value of the anomalous potential on the ellipsoid with a correction for difference in datum potentials.

Table 4 presents the geoid height values using the two evaluation procedures described above and the geoid height differences. The geoid heights obtained from the FORTRAN program refers to the GRS80 reference system.

Table 4. Geoid heights and Geoid height differences

\begin{tabular}{lccc}
\hline Station & $\mathbf{N}^{\text {EGM2008 }}(\mathbf{m})$ & $\mathbf{N}^{\text {GPS/ev }}(\mathbf{m})$ & $\mathbf{N}^{\text {EGM2008 }}(\mathbf{m})-\mathbf{N}^{\text {GPS/lev }}$ \\
\hline JINJA & -14.269 & -13.566 & -0.703 \\
71Y97 (Kampala) & -13.324 & -12.628 & -0.696 \\
KASESE & -10.461 & -9.484 & -0.977 \\
KIBOGA & -13.760 & -13.185 & -0.575 \\
MBARARA & -11.854 & -10.813 & -1.041 \\
MUBENDE & -13.237 & -12.564 & -0.673 \\
RHINO CAMP & -14.930 & -13.578 & -1.352 \\
\hline
\end{tabular}

Geoid heights obtained through a combination of GPS LevellingGPS Levellingheights at the seven GPS LevellingGPS Levellingstations were compared against their respective values synthesized from EGM2008. Table 5 presents the validation results. 
Table 5. Statistics of the Geoid height differences

\begin{tabular}{cccc}
\hline Minimum (m) & Maximum (m) & Mean (m) & Standard Deviation (m) \\
\hline-0.575 & -1.352 & -0.860 & 0.255 \\
\hline
\end{tabular}

There exists a bias (datum offset) of $\sim 0.860 \mathrm{~m}$ between the GPS-levelling derived geoid heights and the geoid heights determined using the gravity model EGM2008. The standard deviation of $0.255 \mathrm{~m}$ gives a measure of the level of agreement between the geoid and GPS Levelling.

The mean value may represent the difference between Uo (potential on the surface of the reference ellipsoid) in this case GRS80 used in the Tg_Schmidt.f synthesis program and Wo (potential at MSL). Using the same method of analysis in Ethiopia, the mean is $-0.978 \mathrm{~m}$ and standard deviation is $0.039 \mathrm{~m}$. This result is similar to the previous study conducted by Bedada (2010), he showed that the combined analysis of EGM2008 with airborne gravity and gravity models of in situ topography and the same mass condensed as a single layer density onto the reference ellipsoid predicts levelled heights at an accuracy of $0.039 \mathrm{~m}$ with much improvement in the mean with the value of $-0.049 \mathrm{~m}$.

Ruling out computational differences since the same methods of analysis were used in Uganda and Ethiopia, the difference in the standard deviations could be due to the quality of the gravity data input during the development of EGM2008. In Ethiopia, EGM08 is dominated by a uniform coverage and high quality internally consistent airborne gravity locally at medium and short wavelengths but by satellite gravity for wavelengths greater than about $400 \mathrm{~km}$ (Bedada 2010). This is in contrast to irregularly distributed and inhomogeneous gravity data available in Uganda.

The datum offset is more interesting. Some of the reasons for this offset include computational effects such as the choice of Wo and Uo, choice of GRS80 for the gravitational reference system and WGS84 for the geometrical reference system and some possibly different tidal conventions. Since a mean sea level which serves as a reference zero height for levelled heights is not identical with the geoid or reference ellipsoid, the choice of the $\mathrm{W}_{0}$ or $\mathrm{U}_{0}$ value affects the vertical separation between the two systems. If the $\mathrm{W}_{0}$ value of geoid is close to the potential of mean sea level compared to $\mathrm{U}_{0}$ value of the reference ellipsoid, choosing $\mathrm{W}_{0}$ is preferred and vice versa. Choosing $\mathrm{W}_{0}=\mathrm{U}_{0}$ is sufficient and doesn't cause any systematic error, it only introduces a constant vertical separation between leveling datum and the geoid (Hipkin 2002). By using the same computation programme for Ethiopia and Uganda, these absolute offset effects are eliminated when comparing the two so that the computed difference $[0.118 \pm 0.255 \mathrm{~m}]$ in datum offset for the two states does tell something about the differences in levelling datums. Because of the $0.255 \mathrm{~m}$ uncertainty, the result is a rather imprecise way of connecting the levelling datums of the two countries but may still be better than nothing. 


\section{Conclusions}

The average standard deviation of the EGM2008 computed geoid heights compared with those computed from the combination of levelled and GPS-based geodetic heights at seven stations reached the level of $0.255 \mathrm{~m}$. This discrepancy can partially be attributed to:

I. The observation errors in levelling. Since there is very little documentation presently available at the Surveys and Mapping Department Entebbe about the historical aspects of the existing levelling network, there is limited information about the accuracy of the levelling results. All documents pertaining to observations and calculations were drafted by the Directorate of Overseas Surveys, and are now kept at the Ordnance Surveys, in Great Britain. Therefore it is not possible to ascertain if the misfit in the geoid heights is due to errors in levelling.

II. Limited gravity data available at the time of the development of EGM2008. According to the International Gravity Bureau, there are 3642 points with gravity data obtained in 1960's distributed through out Uganda. Therefore discrepancies between the geoid heights as presented in Table 3 suggest that there is a possibility of having less gravity data in the Northern part of Uganda (RHINO CAMP) and South Western part of Uganda (MBARARA and KASESE) since the discrepancy in the geoid heights deviates significantly from the geoid height discrepancy of the other 4 points. This however doesn't rule out the effect of topography since rough/undulating terrain as well as thick topographic cover has an effect on the accuracy of the geoid heights computed from the gravity model.

III. The $0.255 \mathrm{~m}$ discrepancy is greatly attributed to the poor and sparsely distributed gravity data set in Uganda.

\section{References}

Aksoy, A., Franke, P., Yalın, D., Bertold, W., 1993. "A Method for Precise Height Difference Determination Based on Trigonometric Leveling”. Prof.Dr.H.Wolf Geodesy Symposium, 3-5 November 1993, İstanbul, (in Turkish).

Banger, G., 1981. Sources of Errors of Precise Levelling, I.U. Journal of Forest Faculty, Vol.31, No: 2, pp 194-207 (in Turkish).

Baykal, O., 1989. Precise levelling technique, I.T.U. Notes of lesson in Graduate school (in Turkish).

Bedada, T.B., An Absolute Geopotential Height System for Ethiopia.2010.165 (Doctor of Philosophy). University of Edinburgh, Edinburgh.

Ceylan, A., 1988. Important Systematic Errors of Precise Levelling, MSc. Thesis, Selcuk Univ., Konya, Turkey, (in Turkish).

Dayoub N., Edwards SJ. and More P., 2012, The Gauss-listing potential value $\mathrm{W}_{0}$ and its rate from altimetric mean sea level and GRACE. J Geod86,681-694. 
Heister H., Lang M., Merry C. L. and Rüther H., 1999. Determination of an Orthometric Height Profile in the Okavango Delta Using GPS Levelling. Proceedings of the FIG Working Week and Survey 1999, Sun City, South Africa, TS 20, B1-B10.

Hipkin R.G., (2002). Defining the geoid by W0=U0: Theory and practice of a modern height system. In: Gravity and Geoid 2002, 3rd Meeting of the International Gravity and Geoid Commission, Thessaloniki, Greece, 367-377.

Kiliçoğlu, A., Direnç, A., Simav, M., Lenk, O., Aktuğ, B., Yildiz H., 2008, Evaluation of the EGM2008 gravity model, in External Quality Evaluation Reports of EGM2008, Newton's Bull. 4, Int. Assoc. of Geod. and the Int. Gravity Field Serv., Toulouse, France.

Kotsakis C, Katsambalos K, Ampatzidis D, Gianniou M 2008. Evaluation of EGM2008 in Greece using GPS and leveling heights. IAG International Symposium on Gravity, Geoid and Earth Observation, Chania, Greece.

Kuntz, E., and Schmitt, G., 1986. Precise Height Determination by Simultaneous Zenith Distances, The Symposium on Height Determination and Recent Vertical Crustal Movements in Western Europe, Hanover, Germany, September 15-19.

Niemeier, W., 1986. Observation Techniques For Height Determination and Their Relation to Usual Height System, The Symposium on Height Determination and Recent Vertical Crustal Movements in Western Europe, Hanover, Germany, September 15-19.

Pavlis NK, Holmes SA, Kenyon SC, Factor JK 2008. An Earth Gravitational Model to Degree 2160: EGM2008, General Assembly of the European Geosciences Union, Vienna, Austria.

Rueger, J.M., and Brunner, F.K., 1982. EDM Height Traversing Versus Geodetic Levelling, The Canadian Surveyor, 36, pp 69-81. 